BULL. AUSTRAL. MATH. SOC.

\title{
HILBERT SPACES HAVE THE BANACH-STONE PROPERTY FOR BOCHNER SPACES
}

\section{Peter Greim}

Let $\left(\Omega_{i}, \Sigma_{i}, \mu_{i}\right)$ be two positive finite measure spaces, $V$ a non-zero Hilbert space, and $1 \leq p<\infty, p \neq 2$. In this article it is shown that each surjective linear isometry between the Bochner spaces $L^{p}\left(\mu_{i}, V\right)$ induces a Boolean isomorphism between the measure algebras $\Sigma_{i} / \mu_{i}$, thus generalizing a result of Cambern's for separable Hilbert spaces.

This Banach-Stone type theorem is achieved via a description of the $L^{p}$-structure of $L^{p}\left(\mu_{i}, V\right)$.

\section{Introduction}

In analogy to the Banach-Stone property [1, p. 142] a Banach space $V$ is said to have the $p$-Banach-Stone property, if for any pair of positive finite measure spaces $\left(\Omega_{i}, \Sigma_{i}, \mu_{i}\right)$ the existence of a surjective linear isometry between the Bochner spaces $L^{P}\left(\mu_{i}, V\right)$ implies that the measure algebras $\Sigma_{i} / \mu_{i}$ are isomorphic. (See [5] for the definition and properties of $L^{p}\left(\mu_{i}, v\right)$.) By Lamperti's extension of Banach's classical result [8] the scalar field $\mathbb{K}$ has the p-Banach-Stone property for

Received 22 September 1982. 
$p \neq 2$. The first vector-valued generalization is due to Cambern who showed that separable Hilbert spaces have this property for $1 \leq p<\infty$, $p \neq 2$ [3] and for $p=\infty$ [4]. Cambern's theorem has been extended to larger classes of separable spaces [9], [6], and [7]; however, for all these Banach-Stone type theorems the separability of $V$ was essential. In this paper we give a generalization of Cambern's theorem for non-separable Hilbert spaces.

THEOREM 1. All non-zero Hilbert spaces have the p-Banach-Stone property $(1 \leq p<\infty, p \neq 2)$.

We shall achieve this result via a description of the ${ }_{L}^{P}$-structure of $L^{P}(\mu, V)$ (see Theorem 2).

As to our method, the proof's in [6], [7], and [9] use some kind of lifting of ${ }_{L}^{p}(\mu, V)$ in order to deal with functions instead of equivalence classes. In this paper we employ a representation of $L^{p}(\mu, V)$ as a space of $L^{p}$-like functions with values in Banach spaces $V_{k}$ that may vary from point to point, a so-called "integral module", introduced by R. Evans. The virtue of this representation is the fact that all norm-functions $k \longmapsto\|x(k)\|$ are continuous, which might turn out to be useful also in the investigations of other geometric relations between $V$ and $L^{p}(\mu, V)$.

\section{An integral module representation}

We need some definitions and notations. From now on let $1 \leq p<\infty$, $p \neq 2$. By $X_{M}$ we denote the characteristic function of a set as well as the corresponding multiplication operator; the constant function with value $v$ is denoted by $v$. In both cases the domains of the functions will be clear from the context. A projection $P$ in a Banach space $X$ is called an $L^{P}$-projection, if

$$
\|x\|^{p}=\|P x\|^{p}+\|x-P x\|^{p}
$$

for all $x \in X$. The set of all $L^{p}$-projections of $X$ is a complete Boolean algebra $[2, \mathrm{p} .11]$ denoted by $\mathbf{P}_{p}(X)$. 
REPRESENTATION THEOREM. Let $(\Omega, \Sigma, \mu)$ be a finite measure space and $V$ a real Banach space, $K$ the Stonean space of the measure algebra $\Sigma / \mu$, and $\Phi$ the Boolean isomorphism of $\Sigma / \mu$ onto the clopen subsets of $K$. Then there is a regular Borel measure $m$ on $K$ satisfying

$$
m(\Phi M)=\mu(M)
$$

for all $M \in \Sigma$ and

$$
m(D)=0
$$

for all measurable nowhere dense $D \subset K$. Furthermore, there is a system $\left(v_{k}\right)_{k \in K}$ of Banach spaces containing $V$ and an embedding

$$
x \mapsto\langle x\rangle
$$

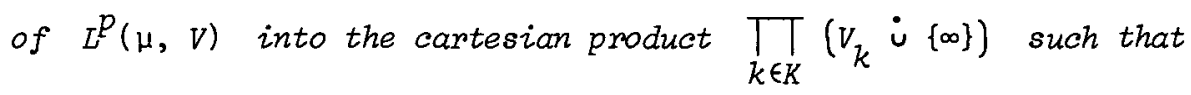

(i) for each $x \in L^{p}(\mu, V)$ the norm function

$$
k \mapsto|x|(k):=\|\langle x\rangle(k)\|
$$

$(\|\infty\|:=\infty)$ is a continuous numerical $I^{p}(m)$-function with $L^{p}$-norm equal to $\|x\|$,

(ii) addition and scalar multiplication on $L^{p}(\mu, V)$, when embedded into the cartesian product, coincide with the m-almost everywhere pointwise operations, precisely: for each $k \in K$ the evaluation mapping

$$
x \mapsto\langle x\rangle(k)
$$

is Zinear on the preimage of $V_{k}$ lobserve that $\langle x\rangle(k) \in V_{k}$ m-almost everywhere since $|x| \in L^{p}(m)$,

(iii) for each $v \in V$ the constant function $v$ on $\Omega$ is mapped onto the constant fronction $v$ on $K$,

(iv) for each $x \in L^{P}(m, V)$ and $M \in \Sigma$,

$$
\left\langle\chi_{M} x\right\rangle=\chi_{\Phi M}\langle x\rangle \quad(0 \cdot \infty:=0) \text {. }
$$

The proof is a simple combination of Theorems 3.7 and 7.3 in [2]. We 
only have to verify the assumption that the set $A$ of characteristic projections $X_{M}(M \in \Sigma)$ in $L^{p}(\mu, V)$ is a complete Boolean algebra of projections in the sense that each increasing net converges strongly to its supremum. This, however, is an immediate consequence of the fact that the set of characteristic projections $x_{M}(M \in \Sigma)$ in the scalar-valued $L^{P}(\mu)$ has this property as it is the whole of $\mathbb{P}_{p}\left(L^{P}(\mu)\right) \quad[2,4.9$ and $1.6]$.

The image of $L^{p}(\mu, V)$ under $\langle\cdot\rangle$ is called an integral module; clearly it is a Banach space isometrically isomorphic to $L^{p}(\mu, V)$.

PROPOSITION. For each $x \in L^{P}(\mu, V)$ there is an open dense subset $U$ of $K$ such that

$$
\begin{aligned}
& \text { (v) }\left.\langle x\rangle\right|_{U} \text { takes its values in } V \text {, } \\
& \text { (vi) }\left.\langle x\rangle\right|_{U} \text { is continuous. }
\end{aligned}
$$

Proof. By ( $i i i)$ and $(i v)$ the simple functions are represented as continuous $V$-valued simple functions, and the latter ones are dense in the integral module. An application of Egorov's theorem shows that $\langle x\rangle$ is an $m$-almost uniform limit of continuous functions. Thus for each $\varepsilon>0$ there is a set $U_{\varepsilon}$ such that $\langle x\rangle$ restricted to $U_{\varepsilon}$ is continuous and $m\left(K \backslash U_{\varepsilon}\right)<\varepsilon$. By the regularity of $m$ we may choose $U_{\varepsilon}$ clopen.

(Observe that an open set and its closure have the same measure.) The union $U$ of all $U_{\varepsilon}^{\prime} ' s$ has the desired properties: its complement has measure zero, so that it cannot contain a non-void clopen subset. As $K$ is 0 -dimensional, this means that $K \backslash U$ has void interior.

\section{The ${ }_{L}^{P}$-structure of ${ }_{L}^{p}(\mu, V)$}

We prove the following theorem.

THEOREM 2. Let $V$ be a non-zero Hilbert space, $(\Omega, \Sigma, \mu)$ a finite positive measure space, $1 \leq p<\infty, p \neq 2$. Then

$$
\mathbf{P}_{p}\left(L^{p}(\mu, V)\right) \cong \Sigma / \mu
$$


Since each surjective isometry $T$ between two Banach spaces $X_{1}$ and $X_{2}$ induces a Boolean isomorphism $\Psi$ of $\mathbf{P}_{p}\left(x_{1}\right)$ onto $\mathbb{P}_{p}\left(x_{2}\right)$ via

$$
\Psi P:=T \circ P \circ T^{-1},
$$

we have an improvement of Theorem 1 as an immediate consequence of Theorem 2.

COROLLARY. For each isometry $T$ of $L^{p}\left(\mu_{1}, V\right)$ onto $L^{p}\left(\mu_{2}, v\right)$ there is a Boolean isomorphism $\Psi$ of $\Sigma_{1} / \mu_{1}$ onto $\Sigma_{2} / \mu_{2}$ such that

$$
\chi_{\Psi M} \circ T=T \circ \chi_{M}
$$

for all $M \in \Sigma_{1}$. That is, the restriction of $T$ to $x_{M} \cdot L^{p}\left(\mu_{1}, V\right)$ is an isometry onto $x_{\Psi M} T^{p}\left(\mu_{2}, V\right)$.

Proof of Theorem 2. Since the characteristic projections $X_{M}$ are trivially ${ }_{L}^{p}$-projections, it remains to show that for each $P \in \mathbf{P}_{p}\left(L^{p}(\mu, V)\right)$ there is an $M \in \Sigma$ such that $P x=X_{M} x$ for all $x \in L^{p}(\mu, V)$. Of course we may assume without loss of generality that the scalars are real. We represent $L^{p}(\mu, V)$ as an integral module as in Section 2 and we show first that $P$ splits into $L^{p}$-projections $P_{k}$ of $V_{k}$, that is,

$$
\langle P x\rangle(k)=P_{k}(\langle x\rangle(k)) \text { almost everywhere }
$$

for all $x \in L^{P}(\mu, V)$. Since the $L^{P}$-projections $P$ and $x_{M}$ commute $(M \in \Sigma)$, we have

$$
\int_{\Phi M}|x|^{p} d m=\left\|\chi_{M} x\right\|^{p}=\left\|P \chi_{M} x\right\|^{p}+\left\|x_{M} x-\chi_{M}^{P x}\right\|^{p}=\int_{\Phi M}\left(|P x|^{p}+|x-P x|^{p}\right) d m
$$

Observe that $\Phi M$ runs through all clopen subsets of $K$. Consequently the continuous (numerical) integrands coincide,

$$
|x|^{p}=|P x|^{p}+|x-P x|^{p} .
$$


In particular $\langle P x\rangle(k) \neq \infty$ for $\langle x\rangle(k) \neq \infty$ and

$$
\langle P x\rangle(k)-\langle P y\rangle(k)=\langle P(x-y)\rangle(k)=0
$$

for $\langle x\rangle(k)=\langle y\rangle(k) \neq \infty$. Thus

$$
P_{k}\langle x\rangle(k):=\langle P x\rangle(k)
$$

is well-defined on $V_{k}=\left\{\langle x\rangle(k) \mid x^{*} \in L^{p}(\mu, V)\right\} \backslash\{\infty\} \quad[2,3.10]$, and (5) says that the projection $P_{k}$ is an $L^{p}$-projection on $V_{k}$. Since $\langle x\rangle(k)=\infty$ only on a set of measure zero, we have (4).

Now we claim $P_{k} \in\{0, I d\}$ for all $k \in K$ (Id the identity operator). Then $B:=\left\{k \mid P_{k}=I d\right\}$ is clopen, for if we choose $v \in V$ with $\|v\|=1$, then

$$
B=\left\{k \mid P_{k} v=v\right\}=\{k|| P v \mid(k)=1\},
$$

and $|P v|$ is a continuous function into the discrete set $\{0,1\}$. Now take $M \in \Sigma$ with $\Phi M=B$. From (ii) and the definition of $B$ we have $m$-almost everywhere, namely for all $k$ with $\langle x\rangle(k) \neq \infty$,

$$
\left|P x-\chi_{M} x\right|(k)=\left\|(P x\rangle(k)-\chi_{B}\langle x\rangle(k)\right\|=\left\|P_{k}(\langle x\rangle(k))-\chi_{B}(k) \cdot\langle x\rangle(k)\right\|=0 ;
$$

that is, $\left\|P x-\chi_{M} x\right\|=0$.

Now let us prove our claim. Hilbert spaces have only the trivial $L^{P}$-projections 0 and Id $[2,1.3]$. So it suffices to verify the parallelogram equality for all components $V_{k}$. Let $\langle x\rangle(k),\langle y\rangle(k)$ be two arbitrary elements of $V_{k}$. By $(v)$, for all $l$ in a dense subset $U$ of $K,\langle x\rangle(Z)$ and $\langle y\rangle(z)$ are elements of the Hilbert space $V$. Thus

$$
|x+y|(z)^{2}+|x-y|(z)^{2}=2\left(|x|(z)^{2}+|y|(z)^{2}\right)
$$

for all $l \in U$, hence, by continuity, everywhere. This shows that $\langle x\rangle(k)$ and $(y)(k)$ satisfy the parallelogram equality, thus completing the proof of Theorem 2 .

REMARK 1. The method of this proof applies to all Banach spaces $V$ where a norm (in-) equality involving only finitely many vectors can be 
shown to imply trivial $L^{P}$-structure, for example all $L^{q}$-spaces $(q \neq p)$.

REMARK 2. The finiteness of the measures in Theorems 1 and 2 is not essential. They hold for $\sigma$-finite measures as well and, if we replace $\Sigma / \mu$ by $P_{p}\left(L^{P}(\mu)\right)$, even for arbitrary positive measures. To see this, observe that each $L^{p}(\mu)$ is an $\tau^{p}$-direct sum $\sum_{i \in I}^{p} L^{p}\left(\mu_{i}\right)$ with finite measures $\mu_{i}$. Evidently we have also $L^{p}(\mu, v)=\sum_{i \in I}^{p} L^{p}\left(\mu_{i}, v\right)$. The fact that a projection in an $l^{p}$-direct sum is an $L^{p}$-projection if and only if its restrictions to the summands are $L^{p}$-projections of the summands $[2,2.6]$ and an application of Theorem 2 show that $\mathbf{P}_{p}\left(L^{p}(\mu, V)\right) \cong \mathbf{P}_{p}\left(L^{p}(\mu)\right)$. For o-finite $\mu$ the latter is $\Sigma / \mu \quad[2,4.9]$.

\section{References}

[1] Ehrhard Behrends, M-structure and the Banach-Stone theorem (Lecture Notes in Mathematics, 736. Springer-Verlag, Berlin, Heidelberg, New York, 1979).

[2] E. Behrends, R. Danckwerts, R. Evans, S. Göbel, P. Greim, K. Meyfarth, W. Müller, $L^{p}$-structure in real Banach spaces (Lecture Notes in Mathematics, 613. Springer-Verlag, Berlin, Heidelberg, New York, 1977).

[3] Michael Cambern, "The isometries of ${ }_{L}^{P}(X, K)$ ", Pacific J. Math. $55(1974), 9-17$.

[4] Mi chael Cambern, "Isometries of measurable functions", Bull. Austral. Math. Soc. 24 (1981), 13-26.

[5] J. Diestel and J.J. Uhl, Jr., Vector measures (Mathematical surveys, 15. American Mathematical Society, Providence, Rhode Island, 1977).

[6] Peter Greim, "The centralizer of Bochner $L^{\infty}$-spaces", Math. Ann. 260 (1982), 463-468. 
[1] Peter Greim, "Isometries and $I^{p}$-structure of separably valued Bochner $L^{p}$-spaces", Measure theory and its applications (Proc. Conf. Sherbrooke, 1982. Lecture Notes in Mathematics. SpringerVerlag, Berlin, Heidelberg, New York, to appear).

[8] John Lamperti, "On the isometries of certain function-spaces", Pacific J. Math. 8 (1958), 459-466.

[9] A.R. Sourour, "The isometries of $L^{p}(\Omega, X)$ ", J. Funct. Anal. 30 (1978), 276-285.

Mathematisches institut,

Freie Universität,

Arnimallee 2-6,

D 1000 Berlin 33,

Germany. 